\title{
Tracking of an underwater visual target with an autonomous surface vehicle
}

\author{
André B. Figueiredo, Bruno M. Ferreira, Aníbal C. Matos \\ INESC TEC, Faculty of Engineering, University of Porto \\ Rua Dr. Roberto Frias, 378 \\ 4200-465 Porto, Portugal
}

\begin{abstract}
This paper presents the development of a first approach to a vision-based target detection. The ultimate objective of this work is to position an autonomous surface vehicle relative to a target. Experiments in a controlled indoor environment were conducted to test the developed system. The experimental results are analyzed and show that the tracking performances achieve errors in the order of a few centimetres.
\end{abstract}

\section{INTRODUCTION}

In opposition to ground robotics, marine robotics is still missing key features to obtain fully autonomous and intelligent robots. The work herein presents the initial design developments and results of a method for totally autonomous tracking and docking of surface and underwater autonomous robots, contributing towards fully automatic missions of marine vehicles. A vision-based method to track and virtually dock an autonomous surface vehicle (ASV) on active markers is presented.

Underwater environments are challenging environments where sensing is generally more limited than on the surface. Therefore, localization methods have been developed and commonly make use of acoustics for large distances. At closer distances, electromagnetic sensing and artificial vision have been complementary solutions. Reliable and effective localization systems usually employ active beacons in the localization process. In this paper, the relative navigation of a marine vehicle with respect to active luminous beacons is exploited. When compared to common, widely used, acoustics-based positioning, artificial vision becomes advantageous for shortrange as it enables a higher feedback control rate and it can provide more precise relative position measurements.

In underwater robotics, relative positioning is an important field where several researchers have been applying considerable efforts. Some examples in vision-based positioning include the works in [1], [2], [3], [4]. In our previous work, the long range relative localization and homing of underwater vehicles was solved [5]. These have provided the vehicle with the capability to reach a given beacon with an error of few meters. This may be satisfactory for a large number of tasks such as vehicle recovery after completion of a mission. Nonetheless, when more precise positioning is required, these methods are of limited use. Following the same trend and based on our previous experience in both theory and practice, the relative positioning of marine vehicles using a camera and visual active markers is explored. The ultimate objective is to implement methods that makes it possible for the vehicles to position themselves precisely with respect to a given reference in order to enable target tracking and automatic docking of both surface and underwater vehicles. Here, a complete method for tracking a reference defined by active markers at short range is proposed.

\section{ARtificial Vision}

Cameras are in general considered cheaper solutions with richer information and providing superior update rates when compared to other commonly used sensors in robotics. Nevertheless, in underwater scenarios, the range of vision sensors is limited because of the typically poor visibility, which is a significant constraint that has to be carefully handled. Although the range constrains the use of vision sensors in underwater environments, cameras are effective sensors for short range situations as they provide meaningful information at relatively high rates. Our current approach considers two artificial directional markers precisely placed on a support frame, which can be either static or mobile. Our system comprises a monocular artificial vision sensor, placed on the vehicle, and two artificial coloured lighting sources (with different colors), mounted on a support frame. In order to estimate the vertical relative distance and the horizontal relative position

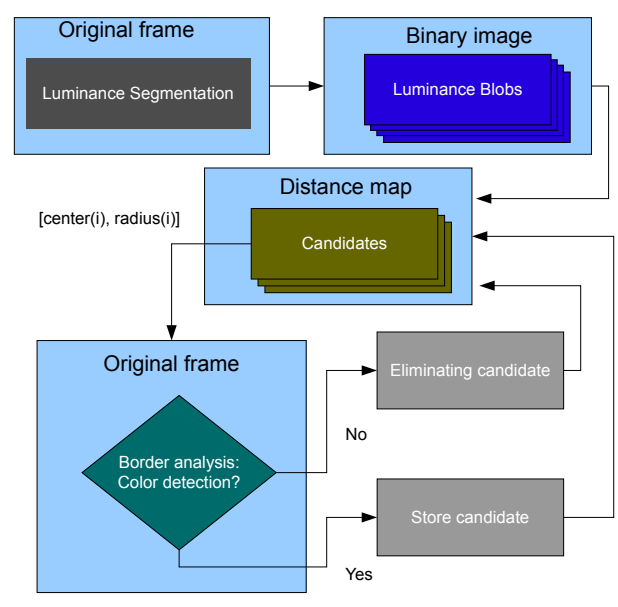

Fig. 1. Beacons visual detection 


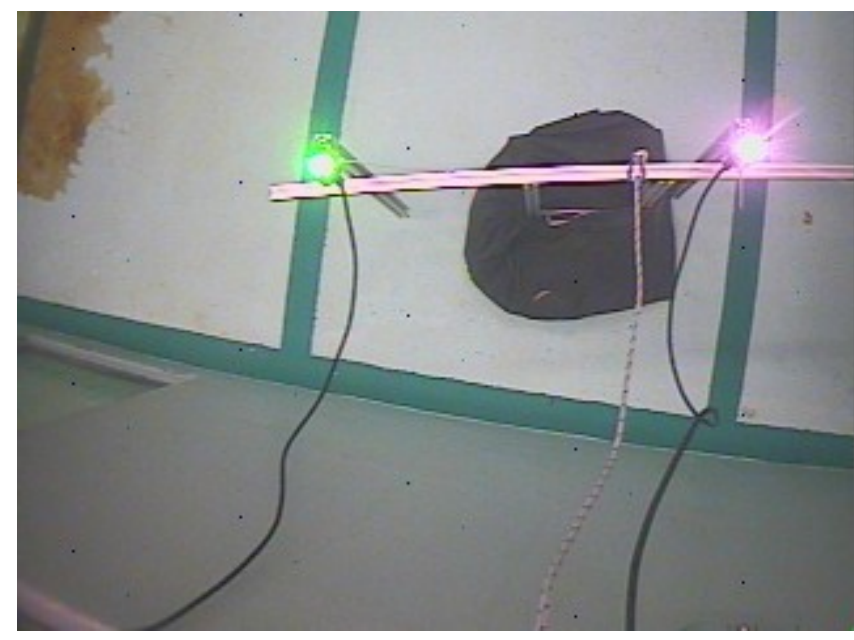

Fig. 2. Acquired image of the two artificial coloured lighting sources mounted on the support platform

with respect to the support platform, the two coloured lighting sources are used as reference markers.

Tracking the coloured light sources in underwater conditions is a challenging problem because it is necessary to robustly distinguish between the desired light sources and other possible sources of illumination. On the other hand, estimating the relative three-dimensional position and estimating the relative orientation between the vehicle and the support frame are also challenging problems. The proposed approach for coloured lighting sources detection is based on a two step image segmentation process. The algorithm diagram is depicted in figure 1. Simply explained, the algorithm makes a luminance segmentation (see figures 2 and 3), from which it extracts relevant pixels where the different reference colors can possibly be identified.

The luminance segmentation may be insufficient to isolate the target beacons. As depicted in figure 3, the processed imaged shows several blobs from which it is necessary to

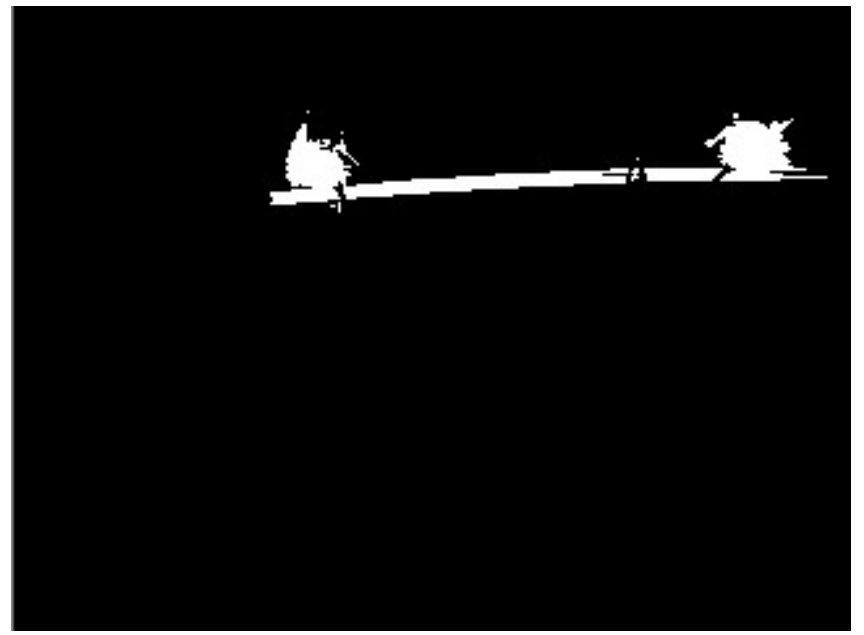

Fig. 3. Luminance image segmentation

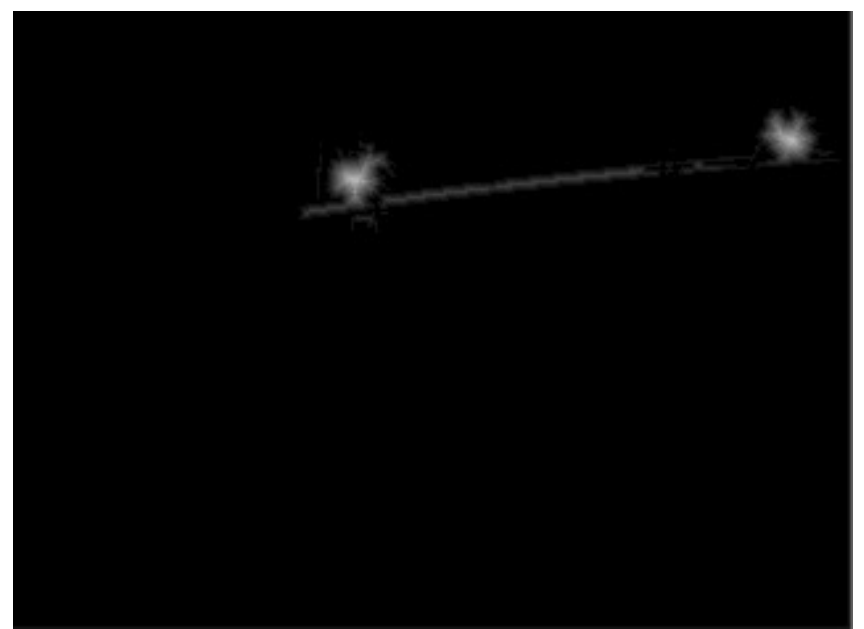

Fig. 4. Distance map image

distinguish the beacons. To do so, a two step procedure is implemented: the first step is to create a distance map and, by using an iterative process, isolate local maxima. This step gives us a center point and a radius for possible candidates to target beacons.

Using the center points and radii from the distance map, the second step of the procedure is to collect samples of pixel RGB values from the border of the identified candidates (at the original frame). The collected samples are then classified according to predefined RGB references: the RGB values are collected in a color vector which are then normalized and compared with normalized color references. If the color is properly classified, the candidate is stored as a valid target and the next candidate is then analysed. In case the color cannot be classified, the candidate is eliminated from the distance map and the next candidate is analysed.

\section{OPTICAL MEASUREMENTS FOR RELATIVE POSITION}

Assuming that the camera sensor plane is parallel to the scene plane and based on the pinhole imaging theory, the optical measurements are made by means of a geometric approach, as shown in figure 5 .

By knowing the resolution of the camera sensor and the pixel size, the position of each point relative to the center of the image is easily extracted. Since the real fixed distance between the two target beacons is known, it is possible to determine a scale factor, $\lambda$, that will make it possible to scale the measurements from the image to the real scene:

$$
\lambda=\frac{d}{d_{A B}^{\prime}}
$$

It is now possible to compute the horizontal relative position of the camera with regard to the beacons. Taking any arbitrarily chosen point A, close to the beacons (possibly coincident), as reference, the horizontal position is computed as:

$$
\left[\begin{array}{l}
x_{A} \\
y_{A}
\end{array}\right]=\left[\begin{array}{l}
x_{A}^{\prime} \\
y_{A}^{\prime}
\end{array}\right] \cdot \lambda
$$




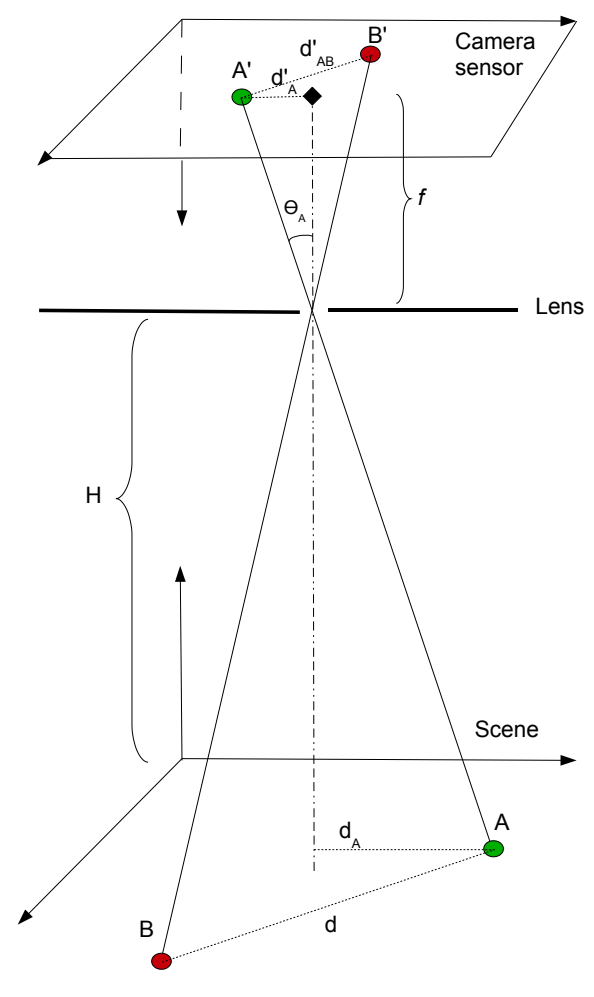

Fig. 5. Optical Measurements

Additionally, in order to compute the vertical position of the camera with regard to the beacons, it is necessary to determine the projection angle, $\theta_{A}$, for a point (taking the point $\mathrm{A}$ as reference). As we know the focal length of our lens, this could be done as follows:

$$
\tan \theta_{A}=\frac{d_{A}^{\prime}}{f}
$$

Considering that the sensor camera plane is parallel to the scene plane, with the calculated projection angles and with the scaled distances we determine the vertical relative distance as:

$$
H=\frac{d_{A}}{\tan \theta_{A}},
$$

where

$$
d_{A}=d_{A}^{\prime} \cdot \lambda .
$$

\section{TARGET TRACKING}

In this work, a control layer that has been previously developed for marine vehicles ([6]) will be further exploited to guide the vehicle over the tracking task. This generic implementation has been successfully governing several underwater and surface vehicles. It currently contemplates two basic layers for dynamics and position stabilization. The former is responsible for driving the velocity to a desired reference which is used by the position guidance law. Field experiments carried over several missions in real environments have already provided very satisfactory results using GPS positional information and inertial measurements. The works in [7], [8] presented the achievable performances.

Using visual feedback in the control and guidance laws, the objective now is to precisely position a vehicle with respect to a virtual point uniquely defined by the two luminous markers. Based on the relative localization data, extracted from the image, as explained in the previous section, a guidance law is derived to make the vehicle track and follow the reference point. This guidance law makes it possible to track both moving and static references. In the static case, the vehicle holds its position while tolerating natural disturbances of marine environments.

Next, a vehicle with surge and yaw degrees of freedom is considered. The notation for the positions and angles is based on the standard NED (north, east, down) referential frame and is borrowed from [9].

\section{A. Control strategy}

The current control implementation includes an inner-outer loop controller. The inner controller stabilizes the vehicle velocities at given, possibly variable, references. This controller makes it possible to "decouple" the vehicle degrees of freedom (DOFs) by actuating in such a way that the different linear and angular velocities are controlled almost independently. This, in turn, implies that simple guidance laws can be developed to generate velocity references and thus abstract from the dynamics level. This lower-level controller was developed in [8] and will not be addressed in this paper. Instead, a more focused attention will be provided to kinematics and position stabilization in the next subsection.

For implementation, the relative positions of the vehicle and of the beacons have to be carefully taken into account. more specifically, particular attention must be given to the behavior to be adopted whenever the beacons are "lost", that is, when the beacons are no longer in the field of view of the camera. This obviously depends on the camera parameters and on the relative distance to the beacons. In this first approach, the position controllers are simply switched off, that is, their output references are set to zero so that no translational nor rotational motion is performed.

\section{B. Guidance law}

Define the relative position of the vehicle with regard to the target (an arbitrarily chosen fixed point close to the beacons) expressed in Cartesian and spherical coordinates as

$$
\tilde{\eta}_{l}=\left[\begin{array}{c}
\tilde{x} \\
\tilde{y} \\
\tilde{z}
\end{array}\right]=\rho\left[\begin{array}{c}
\cos \alpha \cos \beta \\
\sin \alpha \cos \beta \\
-\sin \beta
\end{array}\right],
$$

where $\alpha=\angle(\tilde{x}, \tilde{y})$ and $\beta=\angle\left(\sqrt{\tilde{x}^{2}+\tilde{y}^{2}},-\tilde{z}\right)$, as illustrated in figure 6 , and $\rho=\left\|\tilde{\eta}_{l}\right\|$.

The time derivative of the distance is given as

$$
\dot{\rho}=\frac{1}{\rho} \tilde{\eta}_{l}^{T} \dot{\tilde{\eta}}_{l},
$$




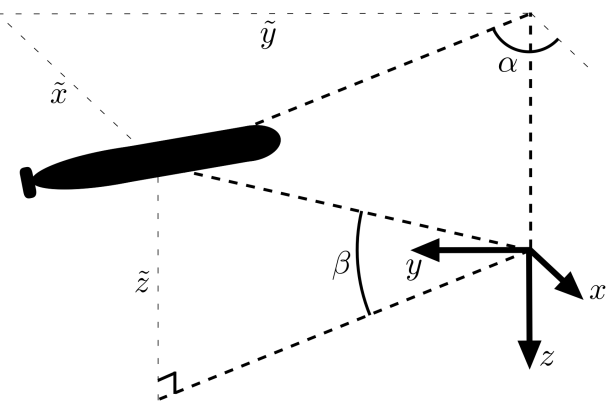

Fig. 6. Coordinates and angles

which can be decomposed as the sum of the components resulting from the vehicle velocity, the drift velocity and the target velocity, as follows:

$$
\dot{\rho}=\vartheta+\vartheta_{D}+\vartheta^{*} .
$$

These components can be seen as the projections of the velocities on the axis resulting from the segment that joins the desired position and the vehicle position. The contribution of the vehicle velocity is given by $\vartheta$, the drift induced velocity component is given by $\vartheta_{D}$ while the contribution of the desired reference position velocity appears in $\vartheta^{*}$. It is assumed that the vertical entries of the target velocity and of the drift velocity are null.

In this paper, the objective is to reduce the horizontal distance of a surface vehicle to the reference point to a predefined offset.

Assuming that the drift velocity and the desired position evolution are known, the aim is to derive a suitable control law that uses $\vartheta$ and its control inputs, to drive the distance between the vehicle and the position to the vicinity of zero. The reason for not driving the vehicle to the exact desired position, is that the $\alpha$ is undefined, and rapidly varies in the presence of disturbances when the vehicle is in the vicinity of the desired position. A closer look at the dynamics of the angles $\alpha$ shows that its time derivative is a function of the inverse of $\rho$. In turn, this implies the non-existence of regular feedback [10]. For this reason, the guidance objective is loosen so that the vehicle reaches the vicinity of the reference (offset), thus enabling the use of regular feedback.

The angular velocity reference is thus chosen as

$$
r^{*}=-K_{\psi}(\psi-\alpha+\pi)+\dot{\alpha},
$$

where $K_{\psi}$ is a constant positive scalars. Assuming that the heading rate is $\psi=r=r^{*}$, then it is possible to conclude that the heading convergence is exponential. Note that the vehicle heading points to the target position projected in the plane where the vehicle lies (surface).

The surge velocity is driven as

$$
u^{*}=\vartheta_{D}+\vartheta^{*}+K_{\rho}\left(\rho_{h}-\delta\right),
$$

where $\delta>0$ is the desired (offset) distance from the reference position, $\rho_{h}=\sqrt{\tilde{x}^{2}+\tilde{y}^{2}}$ is the horizontal component of the distance and $K_{\rho}>0$ is a scalar gain.

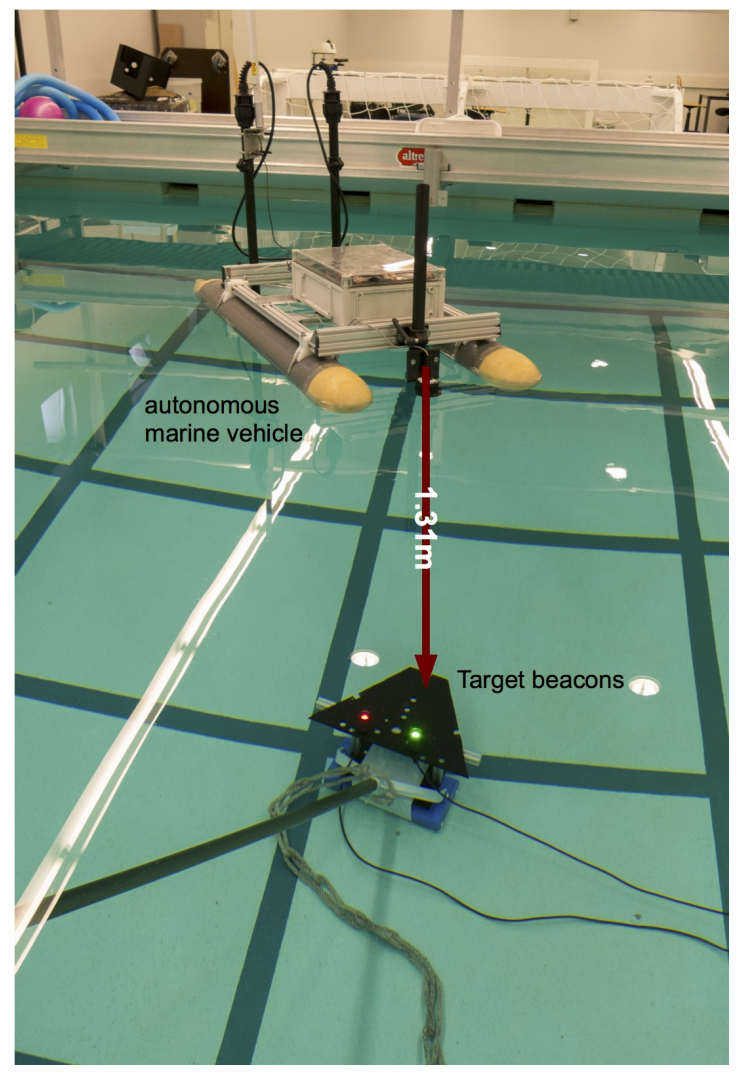

Fig. 7. setup for tests: autonomous surface vehicle and beacons platform

\section{EXPERIMENTAL RESULTS}

Target tracking experiments were conducted to test the system in a tank. The main goals of the experiment were to verify the validity of the developed target detection algorithm and the accuracy of the relative localization and target tracking. The experiments were conducted in a test tank at INESC TEC facilities using an autonomous surface vehicle (ASV) (see Fig. 7) with 1.30 meters of length and 0.60 meters of width. The test tank is 5 meters long, 4 meters wide and 1.6 meters deep. The target platform was placed on the bottom of the tank, within range of view of the camera. The measured vertical distance from the lens of the camera to the beacons was 1.32 meters. The camera was placed in the longitudinal axis of the vehicle, at 0.5 meters from the center of mass $(\mathrm{CM})$ in the bow part. Figure 7 presents the initial conditions.

At a second stage, an experiment with longitudinal disturbance was conducted and for that purpose, a rope was tied to the vehicle with a hanging weight of $500 \mathrm{~g}$. Experiments without disturbance and with disturbance were conducted separately. For both experiments, the tracking offset, $\delta$ (see section IV), was chosen so that the distance of the vehicle (i.e., its $\mathrm{CM}$ ) to the target is equal to the distance of the $\mathrm{CM}$ to the camera. This means that the horizontal position of the camera would be coincident with the horizontal position of the target under perfect tracking.

Figure 8 shows a variance with a maximum amplitude of $7 \mathrm{~cm}$ in the longitudional axis and $7 \mathrm{~cm}$ in the lateral axis. This 


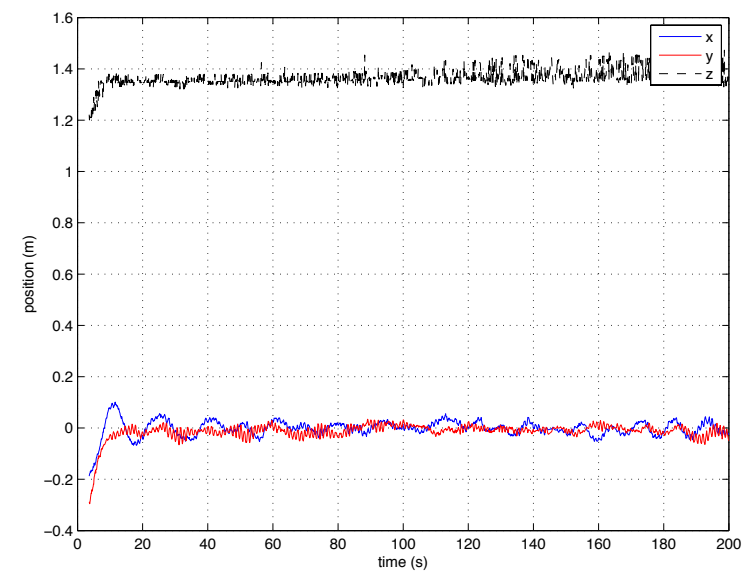

Fig. 8. Relative $\mathrm{x}, \mathrm{y}, \mathrm{z}$ positions without disturbances

variance was caused by a set of factors, including:

- in indoor conditions, no data regarding velocities and absolute position was provided to the controllers. Thus degrading the tracking performance. Moreover, errors related to the actuation model introduce additional undesired effects;

- actuation and motion of the vehicle cause a small disturbances in the water which are then reflected by the tank walls. Such disturbances induce undesired translational and rotational motion;

- noise of the measured relative position mainly originated by: image discretization and unconsidered rotation of the camera plane with regard to the plane containing the beacons.

Despite these disturbances and oversimplified assumption of parallel planes on the optical measurements procedure, results show relatively accurate performances when compared to the vehicles dimensions.

Additionally, an experiment was conducted in order to assess the tracking performances under undesired and neglected effect of an induced force in the longitudinal direction. This experiments is aimed at mimicking the effect of wind and currents in uncontrolled, outdoor, environments. Figure 9 shows the relative position of the vehicle under a longitudinal disturbance. As expected the performance of the tracking controllers degraded to approximately 10 centimetres of error in the longitudinal axis.

\section{CONCLUSIONS}

A first approach to a vision-based target detection, relative localization and target tracking was presented in this paper. In order to easily distinguish artificial features from the scene, active beacons were employed. After acquisition, their relative coordinates in the image are extracted to compute both relative horizontal and vertical positions. This information is then used to feed positioning controllers in order to track an user-defined virtual target.

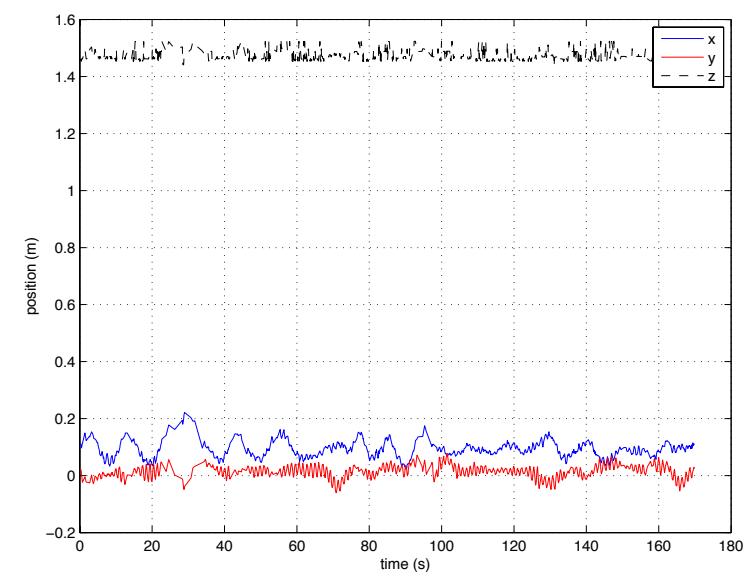

Fig. 9. Relative $x, y, z$ positions with disturbances

Results of this preliminary approach have provided encouraging results in which the relative localization data demonstrated that the vehicle was able to position itself with an error of a few centimetres.

Next developments to be undertaken will include lifting up the assumption of parallel scene and camera planes and relative positioning of underwater vehicles.

\section{REFERENCES}

[1] A. Huster, S. M. Rock, S. P. Boyd, and O. Khatib, "Relative position sensing by fusing monocular vision and inertial rate sensors," 2003.

[2] A. Murarka, G. Kuhlmann, S. Gulati, C. Flesher, M. Sridharan, and W. C. Stone, "Vision-based frozen surface egress: A docking algorithm for the endurance," in AUV, (In Progress) Accepted for Submission to Proceedings UUST09, Conference on Un-manned, Un-tethered Submersible Technology, 2009, pp. 22-25.

[3] J.-Y. Park, B. huan Jun, P. mook Lee, and J. Oh, "Experiments on vision guided docking of an autonomous underwater vehicle using one camera," Ocean Engineering, vol. 36, no. 1, pp. 48 - 61, 2009, ¡ce:title ¿Autonomous Underwater Vehicles;/ce:title $i$ [Online]. Available: http://www.sciencedirect.com/science/article/pii/S0029801808002242

[4] M. Dunbabin, B. Lang, and B. Wood, "Vision-based docking using an autonomous surface vehicle," in ICRA, 2008, pp. 26-32.

[5] B. Ferreira, A. Matos, and N. Cruz, "Single beacon navigation: Localization and control of the mares auv," in OCEANS 2010, 2010.

[6] B. M. Ferreira and A. C. Matos, "Design of a framework for cooperative marine robots," in Proceedings of the StudECE2012 - first PhD conference on Electrical and Computers Engineering, 2012.

[7] N. Cruz, B. Ferreira, A. Matos, C. Petrioli, R. Petroccia, and D. Spaccini, "Implementation of an underwater acoustic network using multiple heterogeneous vehicles," in Oceans, 2012, 2012, pp. 1-10.

[8] B. Ferreira, A. Matos, N. Cruz, and A. Moreira, in Controlo 2014, Porto (to be published).

[9] T. I. Fossen, Guidance and control of ocean vehicles. Wiley, 1994.

[10] E. D. Sontag, "Stability and stabilization: discontinuities and the effect of disturbances, in "Nonlinear analysis, differential equations and control",", 1998. 